\title{
ENERGY EFFICIENCY IN IEEE 802.11 STANDARD WLAN THROUGH MWTDP
}

\author{
Anupam Das ${ }^{1}$, Shikhar Kumar Sarma ${ }^{2}$ \\ ${ }^{1}$ Assistant Professor, Department of Computer Science \& IT, Cotton College, Assam, India adas_arya@rediffmail.com \\ ${ }^{2}$ Professor and Head, Computer and IT, Gauhati University, Assam, India, sks001@email.com
}

\begin{abstract}
The main goal of this work is to achieve the energy efficiency in 802.11 WLAN through minimizing the energy consumption in the network. In this proposed study, we introduced a modification in DCF for enhancing the performance of WLAN and it is achieved by giving a new definition for the DCF function of transmission. Generally, in DCF the way AP transmits for the various nodes is one-way during the process of polling. The proposed function modification for DCF enhances the IEEE 802.11 standard DCF Multi-Way Transmission DCF Protocol (MWTDP) with an improved version MWTDP it gives a low-complexity mechanism by which the active and non-active stations in the BSS save energy during the process of polling. With the inception of MWTDP transmissions are taken place in multi-way the access to the WLAN channel for mobile nodes in the list generated for polling with the SIFS interval whenever the transmission in receiving data packet from AP.
\end{abstract}

Index Terms: MWTDP (Multi-Way transmission DCF Protocol), WLAN (Wireless LAN), DCF(Distributed Coordination Function)

\section{INTRODUCTION}

The main goal of this work is to achieve the energy efficiency in 802.11 WLAN through minimizing the energy consumption in the network. In this proposed study, we introduced a modification in DCF for enhancing the performance of WLAN and it is achieved by giving a new definition for the DCF function of transmission. Generally, in DCF the way AP transmits for the various nodes is oneway during the process of polling. The proposed function modification for DCF enhances the IEEE 802.11 standard

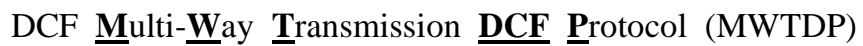
with an improved version MWTDP it gives a lowcomplexity mechanism by which the active and non-active stations in the BSS save energy during the process of polling. With the inception of MWTDP transmissions are taken place in multi-way the access to the WLAN channel for mobile nodes in the list generated for polling with the SIFS interval whenever the transmission in receiving data packet from AP.

\section{METHODOLOGY}

The proposed function modification for DCF enhances the IEEE 802.11 standard DCF $\underline{\text { Multi-Way }}$ Transmission DCF Protocol (MWTDP) with an improved version MWTDP it gives a low-complexity mechanism by which the active and non-active stations in the BSS save energy during the process of polling. With the inception of MWTDP transmissions are taken place in multi-way the access to the
WLAN channel for mobile nodes in the list generated for polling with the SIFS interval whenever the transmission in receiving data packet from AP. The polled mobile nodes or stations can send ACK after the receiving the data packet, by sending a data packet of equal duration of the downlink data packet to the AP. When the mobile nodes listed in the poll do not have data to transmit, it sends only ACK for the data packet or replies with NULL packet irrespective of the AP sends a polled packet because there is no data for the nodes or station. Therefore, the polling operating cost can be minimized when the AP has downlink data for the mobile nodes in the polling list, thus enhancing the utilization of channel. Since the interval of uplink transmissions is fixed by the interval of downlink transmissions, the transmission time of stations in the polling list can be known in advance, from the data packets buffered for these stations at the AP. Thus, the AP can appropriately resolve the interval of a CFP interval to allow an uplink transmission for each station in the polling list. However, not all the mobile nodes in the polling list might have a data packet to transmit. The mobile nodes proposing to leave the polling list because of no more data to send might fail to re-associate with CP. Since DCF is a contention-based protocol, the stations have to compete with all other mobile stations in the same confined area for channel access, and might need several CPs to send the frames of all the nodes caused by the re-association. This phase might degrade the performance of the modified DCF 
incorporating MWTDP. As a solution to this problem, one bit can be taken from the MAC header of the data frame used in CFP, to inform about having more data to transmit. When stations do not have data to send, it sets 0 to the data lists in the MAC header of the last data packet, they have in the queue for transmission; this information helps it to decide for removing these stations from the list prepared by the base station or an AP using a suitable polling scheme. The removal process adopts a very easy and suitable technique for quick elimination and without taking consideration of re-union in CP.

Again when an AP has no data for stations who are in the polling list, it sends a poll packet, in turn the nodes or stations can reply with a data packet of a variable length but with maximum limit (the limit should not cross 2312 bytes). This brings a negative effect on all the other nodes listed in that polling list for the AP. For getting a valid way to overcome this problem is to maintain such information of last downlink data, its duration of each node of the polling list. Again the node should maintain a log table to get the knowledge of the duration of uplink data packet of the APs associated with them.

So, whenever a node gets a poll packet without receiving a data packet, it sends an uplink data packet but having equal duration of last uplink data packet. From this activity of the node, the AP can estimate a proper duration of a CFP interval. It then compare the log table with its own based on the log table containing the information of downlink packets and the polling list.

Sometimes, below performance of the nodes is inevitable as we cannot give $100 \%$ guarantee that under MWTDP the nodes will get greater duration of uplink as compared to its downlink duration as the uplink packet must be fixed by the downlink packet to determine CFP duration. But an approach is incorporated in MWTDP, inserting a sequence of 0's at the end of the uplink packet to first get the equal duration of downlink packet. This also undermine the utilization of channel, but the modification of DCF in MWTDP can provide a precise CFP for the next by doing a comparatively best estimation for the AP and thus AP saves energy during the polling scheme for the nodes. On the completion of CFP intervals estimations, the AP updates the CFP_max duration field. So, all the nodes accordingly update their NAVs with the new CFP duration, when the beacon is transmitted.

Polling nodes and the possible polled nodes monitor the time elapsed from the beacon to ACK during uplink data packet and quickly returns to the doze state until the end of CFP. The time elapsed and the duration of CFP_max, the nodes calculate the sleep period and set it wake up time after receiving the ACK. The wake up time is set to the beginning of CP.

In channel, collisions and errors may take place, but during collision and errors the AP waits for DIFS and sends data packet to the next node which is in the polling list. The nodes having bad conditions due to the collisions and errors, they may stay awake for the CFP, and consume unnecessary some energy. Also the CFP duration changes and in turn the nodes remain in the doze state and keep in this state until the $\mathrm{CP}$ initiated again. Hence, potential instability occurs and to deal with this, the AP does not announce a new CFP until duration of CFP_max expires. This allows the Polled nodes to wake up and get the next CFP repetition interval. Communications among nodes through AP involving some nodes in Polled nodes gets frozen during CFP interval and data packets are buffered at the concerned AP until the duration of CFP_max seizes.

The modified DCF with the MAC enhancement along with the energy consumption is briefed in the figure-3.2(h) It is distinct that the last station in the polled list consumes higher energy when the number of active stations or nodes are large. DCF uses round robin technique for preparing polling sequence and nodes are assigned association ids (aid)s of accepted nodes in ascending order. To minimize the energy consumption a new approach is adopted i.e. Cyclic-round robin in MWTDP. These modifications brings performance enhancement in the network along with less impact on channel utilization.

\section{ANALYSIS}

TABLE-1 END TO END DELAY IN CLP AND NBEP

\begin{tabular}{|l|l|l|}
\hline Time & $\begin{array}{c}\text { E2E } \\
\text { Delay in } \\
\text { CLP(with } \\
100 \\
\text { nodes) }\end{array}$ & $\begin{array}{c}\text { E2E Delay } \\
\text { in } \\
\text { NBEP(with } \\
100 \text { nodes) }\end{array}$ \\
\hline 0.0 & 0 & 0 \\
\hline 0.5 & 0.02 & 0.01 \\
\hline 0.8 & 0.06 & 0.03 \\
\hline 0.85 & 0.09 & 0.04 \\
\hline 0.9 & 0.16 & 0.07 \\
\hline 1.0 & 0.25 & 0.08 \\
\hline 1.2 & 0.29 & 0.099 \\
\hline 1.4 & 0.33 & 0.109 \\
\hline 1.6 & 0.38 & 0.12 \\
\hline 1.8 & 0.41 & 0.13 \\
\hline 2.0 & 0.46 & 0.15 \\
\hline & & \\
\hline
\end{tabular}


TABLE-2 PACKET DELIVERY RATIO

\section{IN CLP AND NBEP}

\begin{tabular}{|l|l|l|}
\hline Time & $\begin{array}{l}\text { PDR in } \\
\text { CLP(with } \\
100 \\
\text { nodes) }\end{array}$ & $\begin{array}{l}\text { PDR in } \\
\text { NBEP(with } \\
100 \text { nodes) }\end{array}$ \\
\hline 0.0 & 0 & 0 \\
\hline 0.5 & 27 & 45 \\
\hline 0.8 & 58 & 84 \\
\hline 0.85 & 79 & 105 \\
\hline 0.9 & 96 & 135 \\
\hline 1.0 & 155 & 175 \\
\hline 1.2 & 160 & 210 \\
\hline 1.6 & 178 & 230 \\
\hline 1.9 & 188 & 244 \\
\hline 2.2 & 202 & 261 \\
\hline
\end{tabular}

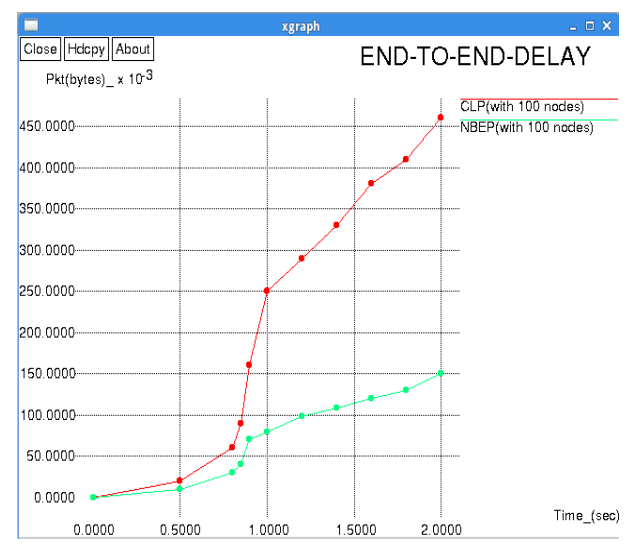

FIGURE-1 END TO END DELAY IN CLP AND NBEP

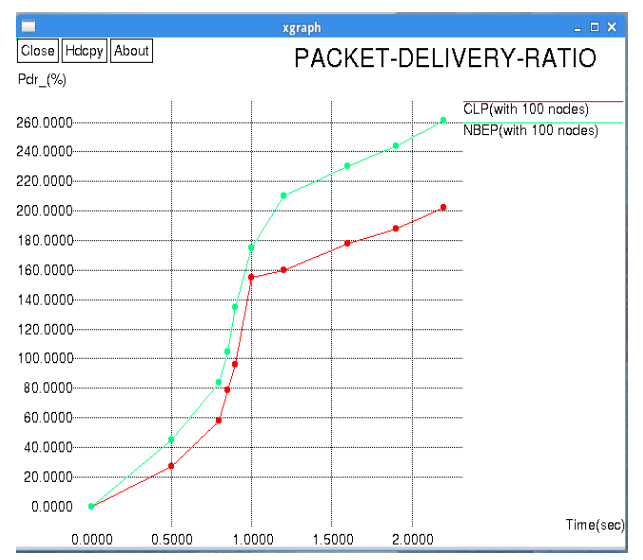

FIGURE-2 PACKET DELIVERY RATIO IN CLP AND NBEP
TABLE-3 PACKET LOST

IN CLP AND NBEP

\begin{tabular}{|l|l|l|}
\hline Time & $\begin{array}{c}\text { Packet lost } \\
\text { in CLP } \\
\text { (with 100 } \\
\text { nodes) }\end{array}$ & $\begin{array}{c}\text { Packet lost } \\
\text { in NBEP } \\
\text { (with 100 } \\
\text { nodes) }\end{array}$ \\
\hline 0 & 0 & 0 \\
\hline 5 & 1 & 1 \\
\hline 10 & 1.16 & 1.01 \\
\hline 15 & 1.6 & 1.3 \\
\hline 20 & 1.9 & 1.5 \\
\hline 25 & 2.2 & 1.7 \\
\hline 30 & 2.8 & 1.91 \\
\hline 35 & 3.1 & 2.01 \\
\hline 40 & 3.3 & 2.03 \\
\hline 45 & 3.7 & 2.2 \\
\hline 50 & 4.0 & 2.33 \\
\hline
\end{tabular}

TABLE-4 ENERGY CONSUMPTION IN CLP AND NBEP

\begin{tabular}{|l|l|l|}
\hline Time & $\begin{array}{c}\text { Energy } \\
\text { Spent in } \\
\text { CLP } \\
\text { (with 100 } \\
\text { nodes) }\end{array}$ & $\begin{array}{c}\text { Energy } \\
\text { Spent in } \\
\text { NBEP } \\
\text { (with 100 } \\
\text { nodes) }\end{array}$ \\
\hline 0 & 1000 & 1000 \\
\hline 25 & 952 & 992 \\
\hline 50 & 895 & 923 \\
\hline 75 & 812 & 876 \\
\hline 100 & 715 & 815 \\
\hline 150 & 612 & 762 \\
\hline 200 & 512 & 727 \\
\hline 250 & 490 & 687 \\
\hline 300 & 471 & 652 \\
\hline 350 & 458 & 618 \\
\hline 400 & 431 & 578 \\
\hline
\end{tabular}




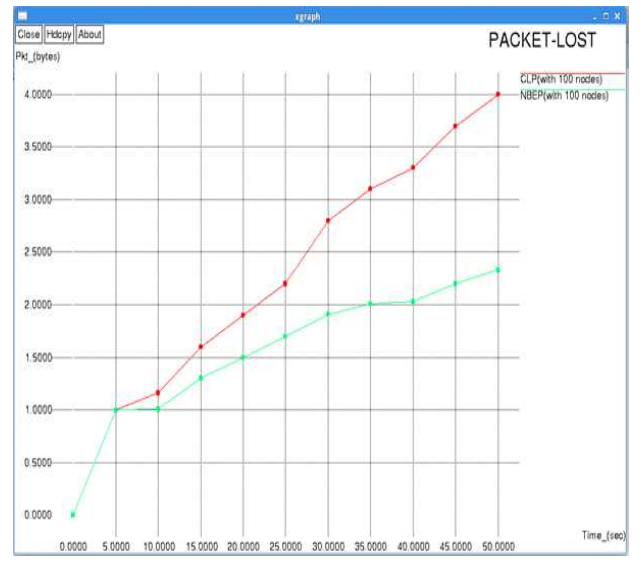

FIGURE-3 PACKET LOST

IN CLP AND NBEP

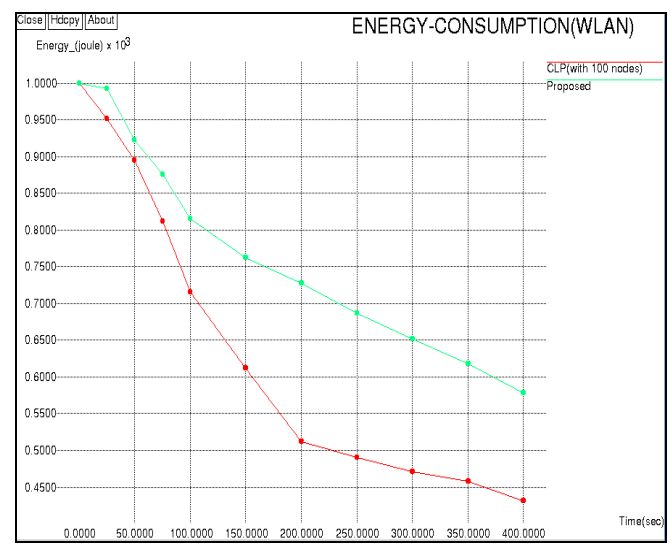

FIGURE-4 ENERGY CONSUMPTION

IN CLP AND NBEP

TABLE-5 SUMMARY OF ENERGY CONSERVATION \& ENERGY SPENDING (WITH 100 NODES)

\begin{tabular}{|l|l|c|c|c|c|c|}
\hline Time & $\begin{array}{c}\text { Energy } \\
\text { Conserved } \\
\text { (Existing } \\
\text { System) }\end{array}$ & $\begin{array}{c}\text { Energy } \\
\text { Conser } \\
\text { ved } \\
\text { (Propo } \\
\text { sed } \\
\text { System } \\
\text { ( }\end{array}$ & $\begin{array}{c}\text { Energy } \\
\text { Spent } \\
\text { in } \\
\text { Existing } \\
\text { system }\end{array}$ & $\begin{array}{c}\text { Energ } \\
\text { y } \\
\text { spent } \\
\text { in } \\
\text { propo } \\
\text { sed } \\
\text { vvste }\end{array}$ & $\begin{array}{c}\text { Energy } \\
\text { spending } \\
\text { (in \%) } \\
\text { Proposed }\end{array}$ & $\begin{array}{c}\text { Ener } \\
\text { gy } \\
\text { spen } \\
\text { ding } \\
\text { (in } \\
\%) \\
\text { Evist }\end{array}$ \\
\hline 0 & 1000 & 1000 & 0 & 0 & 0 & 0 \\
\hline 25 & 952 & 992 & 48 & 8 & 0.8 & 4.8 \\
\hline 50 & 895 & 923 & 105 & 77 & 7.7 & 10.5 \\
\hline 75 & 812 & 876 & 188 & 124 & 12.4 & 18.8 \\
\hline 100 & 715 & 815 & 285 & 185 & 18.5 & 28.5 \\
\hline 150 & 612 & 762 & 388 & 238 & 23.8 & 38.8 \\
\hline 200 & 512 & 727 & 488 & 273 & 27.3 & 48.8 \\
\hline 250 & 490 & 687 & 510 & 313 & 31.3 & 51.0 \\
\hline 300 & 471 & 652 & 529 & 348 & 34.8 & 52.9 \\
\hline 350 & 458 & 618 & 542 & 392 & 39.2 & 54.2 \\
\hline 400 & 431 & 578 & 569 & 422 & 42.2 & 56.9 \\
\hline
\end{tabular}

When the MWTDP is again tested with the same test bed the results we got to evaluate the performance of the WLAN are shown in the following graphical results. All the performance metrics are shown satisfactory improvement over the CLP and also our earlier NBEP-model[1].

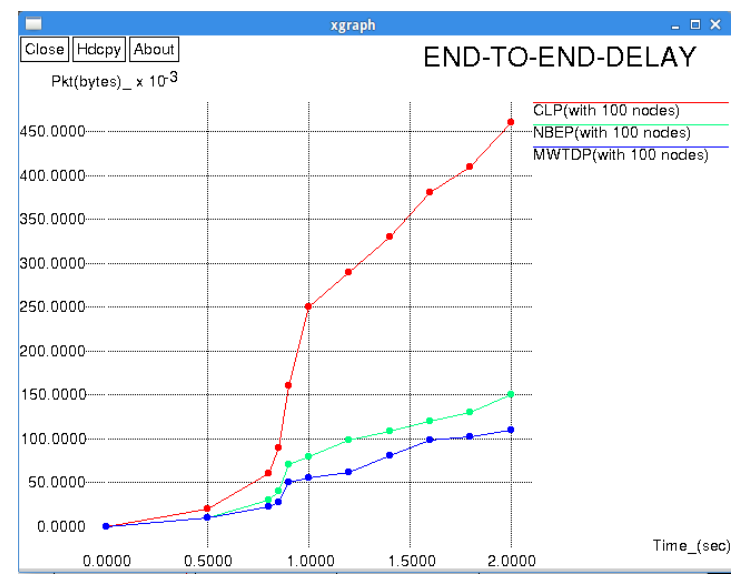

FIGURE-5 END TO END DELAY IN CLP , NBEP AND MWTDP

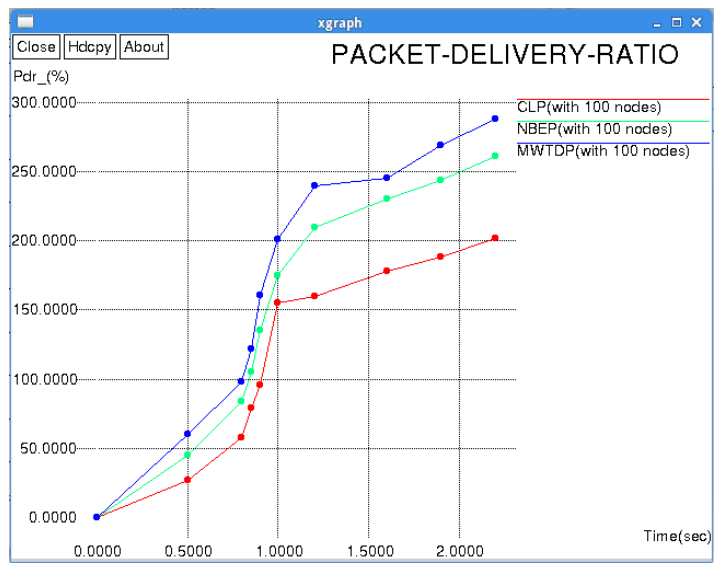

FIGURE-6 PACKET DELIVERY RATIO IN CLP , NBEP AND MWTDP

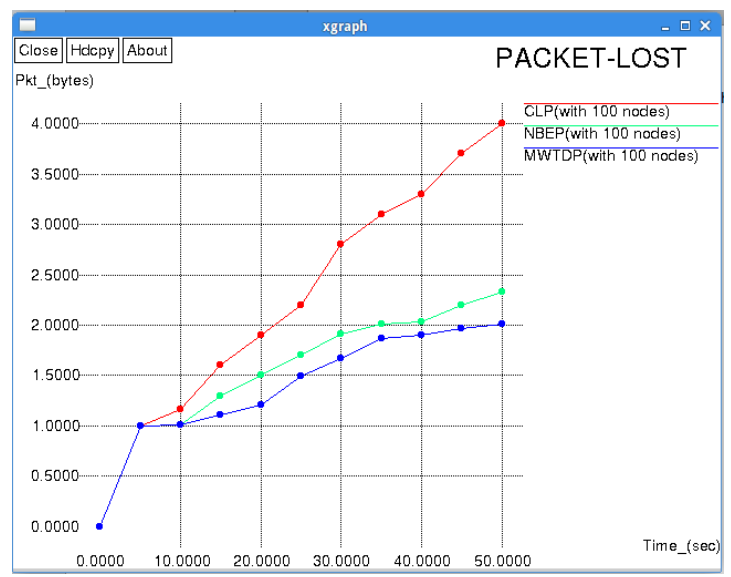

FIGURE-7 PACKET LOST

IN CLP, NBEP AND MWTDP 


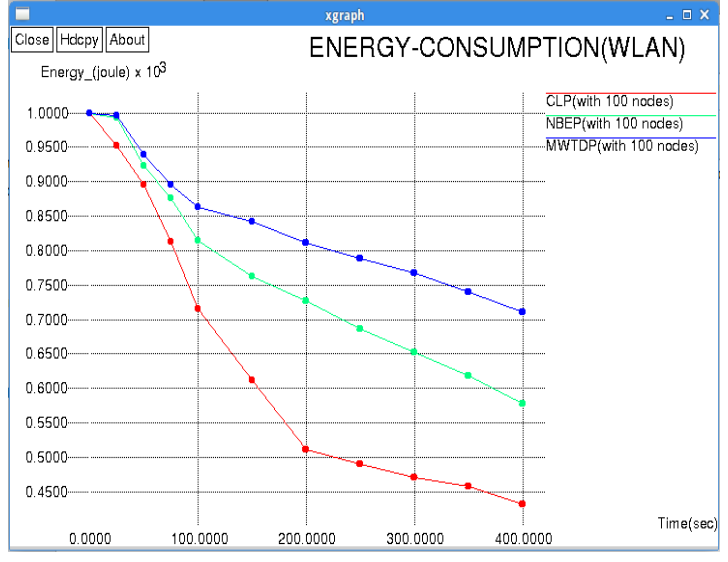

FIGURE-7 ENERGY CONSUMPTION

IN CLP, NBEP AND MWTDP

\section{CONCLUSIONS}

In the above discussion, Energy Conservation has been analyzed with the help of the graph generated by the ns 2 and thus get conclusive figures of energy spending in percentage. In the graph it is seen at the very outset of the time interval the energy in both the systems, i.e. in the existing and proposed is considered as 1000 joule. The result indicates the betterment of the system with the help of the proposed model over the existing system. After $25 \mathrm{sec}$ the energy conserved in existing system is 992 joule as compared to 952 joule in the existing system. In percentage the $0.8 \%$ energy spending is in the proposed system and $4.8 \%$ energy spending in the existing system. Hence very less amount of energy is spent in the proposed system as compared to the existing system. After $50 \mathrm{sec}$ the energy conserved in existing system is 923 joule as compared to 895 joule in the existing system. In percentage the $7.7 \%$ energy spending is in the proposed system and $10.5 \%$ energy spending in the existing system. Hence very less amount of energy is spent in the proposed system as compared to the existing system. After $75 \mathrm{sec}$ the energy conserved in existing system is 876 joule as compared to 812 joule in the existing system. In percentage the $12.4 \%$ energy spending is in the proposed system and $18.8 \%$ energy spending in the existing system. Here is also a significant less amount of energy is spent in the proposed system as compared to the existing system. After $100 \mathrm{sec}$ the energy conserved in existing system is 815 joule as compared to 715 joule in the existing system. In percentage the $18.5 \%$ energy spending is in the proposed system and $28.5 \%$ energy spending in the existing system. At this stage also a less amount of energy is spent in the proposed system as compared to the existing system. After $150 \mathrm{sec}$ the energy conserved in existing system is 762 joule as compared to 612 joule in the existing system. In percentage the $23.8 \%$ energy spending is in the proposed system and $38.8 \%$ energy spending in the existing system. Hence very less amount of energy is spent in the proposed system as compared to the existing system. Finally, after time period, 200 seconds, the energy conserved in existing system is 727 joule as compared to 512 joule in the existing system. In percentage the $27.3 \%$ energy spending is in the proposed system and $48.8 \%$ energy spending in the existing system. Hence very less amount of energy is spent in the proposed system as compared to the existing system.

After increasing the network size, i.e. instead of 50 nodes we used 100 nodes and again analyzed the graphs generated by NS2. It is significantly shown the better results in NBEP with MWTDP model as compared to CLP.

We further implemented the NBEP with a new approach and named the model as MWTDP and the results we got are fairly improved. The analytical graphs are shown in figures-5 to figure-7. In all the graphs the QoS performance metrics of WLAN are shown better results as compared to CLP and as well as our earlier model NBEP[1].

\section{ACKNOWLEDGEMENT}

I sincerely thank my supervisor Prof. Shikar Kumar Sarma for his constant encouragement for carrying out this study. He always gives me valuable guidance technical as well as conceptual whenever I face any problem in my research work.

\section{REFERENCES}

[1]. A Study on Energy Consumption in WLAN and Improving its efficiency through NBE-algorithm by A Das \& Prof. Shikhar Kr. Sarma

[2]. Energy Efficiency of Ad hoc Wireless Networks with selfish users by Vikrma Srinivasan, Pavan Nagcahlli.

[3] Energy Efficiency Analysis of IEEE 802.11 DCF with variable Packet length was carried out by Bo Gao, Yuhang Yang Huiye Ma

[4] Modeling Energy Efficiency Secure Wireless Neyworks using Network Simulation By Ramesh Puri and Piyush Misra. 


\title{
BIOGRAPHIES
}

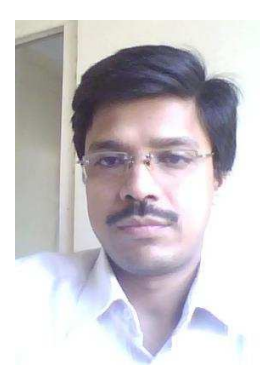

\begin{abstract}
Anupam Das is a $\mathrm{PhD}$ scholar and working as an Assistant Professor for last 7 years. He has published more than 5 research papers. He has also published 4 Books. $\mathrm{He}$ did masters in Mathematics and Computer Science and also completed M. Phil in Computer Science.
\end{abstract}

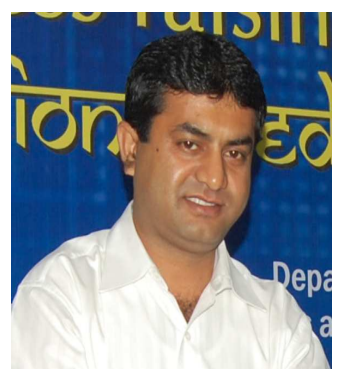

Professor Shikhar Kr Sarma is currently working as Director in the Institute of Science and Technology of Gauhati University. He has the distinct contributions in the arena of research publications. In his credit he has 15 Journals, 7 Seminar volumes, 24 Conference volumes, 1 E-Book and 1 Book (Edited). 